\title{
Aharonov-Bohm Olayı ve Monopol
}

\author{
Çılga MİSLİ, Oktay YILMAZ* \\ Çanakkale Onsekiz Mart Üniversitesi, Fen Edebiyat Fakültesi Fizik ABD, Çanakkale
}

\section{Özet}

David Bohm ve doktora öğrencisi Yakir Aharonov 1959 yılında Aharonov-Bohm (AB) olayını keşfettiler. Daha sonra AB olayı 1986 yılında A. Tonomura ve arkadaşları tarafindan deneysel olarak doğrulanarak daha çekici bir hale gelmiştir (Tonomura ve ark., 1982, 1986). AB olayının anlaşılması ile kuramsal fiziğin temelleri ve $A B$ olayı ile ilgili başka fiziksel olay ve kavramlar daha iyi anlaşılmaktadır.

$\mathrm{Bu}$ çalışmada, Aharonov-Bohm olayı incelenip tartışılmaktadır (Aharonov ve Bohm, 1959). AB olayının sonucunda, kuantum mekaniğinde elektromagnetik (EM) potansiyellerin ilginç özellikleri ortaya çıkmakta ve ayrı bir önem kazanmaktadır. Klasik mekaniğe göre, alanlar yüklü parçacıkların kendisi ile direk etkileşmektedir fakat Aharonov-Bohm olayına göre etkiyen hiçbir alan olmasa bile yüklü parçacıkların elektromagnetik potansiyellerle etkileştiği anlaşılmaktadır.

Ayar dönüşümleri altında Schrödinger dalga denkleminin değişmez (invaryant) kalması, dalga fonksiyonun dönüşmesini gerektirir. Dönüşen dalga fonksiyonu bir faz çarpanı içerir ve bu faz, elektromagnetik potansiyellere bağlıdır. Önerilen Aharonov-Bohm deneyinde hiç bir alanla etkileşmeyen fakat sadece elektromagnetik potansiyeller ile etkileşen elektronlar girişim deseninde kaymaya neden olur. Bunun sonucunda, faz kayması, magnetik ak1, magnetik tek-kutup (monopole) kuantizasyonu gibi önemli sonuçlar elde edilir. Ayrıca, yarı-klasik yöntemle de magnetik tek-kutup ve elektromagnetik açisal momentum kuantizasyonu yapılabilmektedir (Sakurai, 1994; Jackson, 1999) .

Ayar dönüşümünün fiziksel anlamı ve vektör potansiyel hesaplarından sonra bir sarmal (selenoid) ile etkileşen yüklü parçacıkların saçılma genliği ve diferansiyel tesir kesit hesapları yapılarak, sonuçları tartışılmaktadır.

Anahtar sözcükler: Aharonov-Bohm, Ayar Dönüşümü, Dirac Kuantizasyonu, Saçılma, Tek-kutup (Monopol). 


\title{
Aharonov-Bohm Effect and Monopole
}

\begin{abstract}
David Bohm and his Ph.D. student Yakir Aharonov discovered Aharonov-Bohm (AB) effect in 1959. Then AB effect has been confirmed by experiment which was done by A. Tonomura and his colleagues and then it became more attractive (Tonomura and et al., 1982, 1986). By understanding AB effect, fundamental of theoretical physics and other physical phenomena which is related to $\mathrm{AB}$ effect, are better understood.
\end{abstract}

In this study, $A B$ effect is discussed and analyzed here (Ahoronov and Bohm, 1959). As a result of $A B$ events, an interesting properties and significance of electromagnetic potentials are appeared in quantum theory. In classical mechanics, charged particles are interacted with the electromagnetic field itself directly. But according to $\mathrm{AB}$ effect, even if there is no electromagnetic fields, charged particles are interacted with the electromagnetic potentials.

Schrödinger equation does not change under the gauge transformation, then wave function needs to be transformed as we know that wave function can be multiplied by a phase factor. This phase factor is depends on electromagnetic potentials and it causes quantum interference patern to shift has been observed by $A B$ experiment, then we see that charged particles are interacted with electromagnetic potentials (not fields). As a result of these kind of events leads to phase shift, magnetic flux, magnetic monopole, quantization etc. Additionally, magnetic monopole and electromagnetic angular momentum could be quantized by using semi-classical methods (Sakurai, 1994; Jackson, 1999).

After the meaning of the gauge symmetry and calculation of vector potential, we disscus the scattering of charged particles on the selenoid (Dirac string), scattering amplitude and differantial cross section.

Keywords: Aharonov-Bohm, Dirac Quantization, Gauge Transformations, Scattering, Monopole. 


\section{Giriş}

David Bohm ve doktora öğrencisi Yakir Aharonov 1959 yılında Aharonov-Bohm (AB) olayını keşfettiler (Aharonov ve Bohm, 1959). Sayısız çalışmaları ve bilime katkılarıyla tanınan D. Bohm 1990 yılında Royal Society üyeliğine seçilmiş ve 1991 yılında, Y. Aharonov ile birlikte Elliot Cresson Altın Madalyasını almıştır.

Elektromagnetik (EM) alanların etkisindeki yüklü parçacıkların hareketinin kuantum mekaniğiyle nasıl anlatılacağı ciddi bir araştırma gerektirmektedir. Deneylerle uyumlu olduğu gözlenen Aharonov-Bohm olayı fizikçilerin daha çok ilgisini çekmiş ve A-B olayıyla ilgili başka fiziksel olay ve kavramların daha iyi anlaşılabileceğini düşünmüşlerdir.

$\mathrm{Bu}$ çalışmada, kuramsal fiziğin önemli bir parçası ve fiziğin her alanıyla ilişkisi olan Aharonov-Bohm olayı tartışılmaktadır.

\subsection{Klasik Limit}

EM alanındaki yüklü parçacığın Lagrange fonksiyonu potansiyellere bağlıdır. Eylemin $\delta S=0$ varyasyon (minimum eylem ilkesi) hesabından Euler-Lagrange denklemleri ve buradan parçacığın klasik davranışı hakkında bilgiler elde edilebilir. Lagrange fonksiyonu bilindiği için Hamiltonyen de bilinir dolayısıyla kuantum mekaniksel yöntemle ilgilenilen sistemdeki potansiyeller amaca uygun bir şekilde değiştirilerek incelenebilir. Hamiltonyenin operatör formu Schrödinger dalga denklemidir ve özel limit (asimptotik) durumu, HamiltonJacobi denklemini verir. Schrödinger dalga denkleminin çözümü $\psi(x, t)=e^{i S(x, t) / \hbar}$ önerilerek ve $\left|\frac{i \hbar s^{\prime \prime}}{S^{\prime 2}}\right| \ll 1$ yaklaşımı (WKB) ile Hamilton-Jacobi denklemi elde edilir (Schiff, 1949). Bunun sonucunda $H(x, p)=\frac{p^{2}}{2 m}+V(x)$ Hamiltonyen ve $p=\frac{\partial S}{\partial x}$ kanonik (eşlenik) momentum elde edilir. Burada $S(x, t)$ fonksiyonu iki boyutlu uzayda bir eğriyi ve üç boyutlu uzayda bir yüzeyi temsil eder. Momentum vektörü, $S$ yüzeyine dik ve klasik parçacığın izlediği yörünge boyuncadır (Schiff, 1949). Kuantum mekaniğinden klasik mekaniğe bir yaklaşımla geçiş yapılabildiği için, bu tür çözümlere yarı-klasik çözümler denir. Bilinen klasik mekanik hesaplariyla Lagrange fonksiyonu $L=p \dot{x}-H$ ve $S=\int L d t$ eylem (action) bulunur. Eğer $S(x, t)=S(x)-E t$ gibi değişkenlerine ayrılabilirse, Hamiltonyen $H(x, p)=$ $E$ ve kararlı durum $\psi(x, t)=e^{i S(x) / \hbar} e^{-i E t / \hbar}$ çözümü bulunur. Burada $E$ enerji, $|\psi(x, t)|^{2}$ olasılık yoğunluğu olarak tanımlanır ve zamandan bağımsız sabit bir sayıdır. Eylemin $\delta S=0$ varyasyon hesabindan

$$
\frac{d}{d t}\left(\frac{\partial L}{\partial \dot{x}_{j}}\right)-\frac{\partial L}{\partial x_{j}}=0 \quad p_{j}=\frac{\partial L}{\partial \dot{x}_{i}}=m \dot{x}_{j}+q A_{j}
$$

Euler-Lagrange denklemleri ve $p_{j}$ kanonik momentum elde edilir (Goldstein, Poole ve Safko, 2002). EM alanda hareket eden yüklü parçacı̆̆ın Lagrange fonksiyonu $L=\frac{1}{2} m \dot{x}_{i} \dot{x}_{i}+$ $q A_{i} \dot{x}_{i}-q \phi$ verildiğinde

$$
m \ddot{x}_{j}=q E_{j}+q(v \times B)_{j}
$$


Newton'nun hareket yasası elde edilir. Burada $E_{j}=-\frac{\partial A_{j}}{\partial t}-\frac{\partial \phi}{x_{j}}$ elektrik ve $B_{j}=(\boldsymbol{\nabla} \times \boldsymbol{A})_{j}$ magnetik alandır (Jackson, 1999). Hamiltonyen $H=p_{j} \dot{x}_{j}-L$ ifadesinde Lagrange fonksiyonu ve $m \dot{x}_{j}$ kinetik momentum yerine yazılarak, EM alanla etkileşmeyi anlatan

$$
H=\frac{\left(p_{j}-q A_{j}\right)^{2}}{2 m}+q \phi
$$

hamiltonyen bulunur. Böylece sistemin hamiltonyeni bilindiği için, kuantum mekaniğiyle potansiyeller istenilen amaca uygun şekilde değiştirilerek incelenebilir.

\subsection{Ayar Dönüşümü}

Aharonov-Bohm olayı, ayar simetrisinin sonuçlarından biridir ve neden sonuç ilişkisi açısından birbirlerini tamamlarlar. Dalga fonksiyonu bir faz çarpanı ile dönüştügü için ve girişime neden olduğu için bu dönüşüme faz dönüşümü ya da dönüşümler simetriyi koruduğu için ayar simetrisi de denir. AB olayının anlaşılmasıyla ayar teorisi daha anlamlı hale gelmektedir. Schrödinger dalga denkleminin ayar dönüşümleri altında değişmemesi dalga fonksiyonun dönüşmesine neden olur. Dönüşen dalga fonksiyonu; magnetik akı, elektrik ve magnetik yükün kuantizasyonu, girişim deseninde faz kayması ve $\mathrm{AB}$ olayı gibi önemli sonuçlara neden olur (Sakurai, 1994).

Bilindiği gibi SI birim sisteminde ve boşlukta Maxwell denklemleri

$$
\begin{aligned}
& \begin{array}{lll}
\boldsymbol{\nabla} \cdot \boldsymbol{E}=\frac{\rho}{\epsilon_{0}} & \boldsymbol{\nabla} \cdot \boldsymbol{B}=0 \quad \text { (Gauss) }
\end{array} \\
& \boldsymbol{\nabla} \times \boldsymbol{E}=-\frac{\partial \boldsymbol{B}}{\partial t} \quad \text { (Faraday) } \\
& \boldsymbol{\nabla} \times \boldsymbol{B}=\mu_{0} \boldsymbol{j}+\mu_{0} \epsilon_{0} \frac{\partial \boldsymbol{E}}{\partial t} \quad \text { (Ampere-Maxwell) }
\end{aligned}
$$

ile verilir (Jackson, 1999). Burada $\rho$ yük ve $\boldsymbol{j}$ akım yoğunluğu olarak tanımlanır,

$$
\frac{\partial \rho}{\partial t}+\boldsymbol{\nabla} \cdot \boldsymbol{j}=0
$$

yük korunum yasası Maxwell denklemleri içinde kendiliğinden sağlanır. (1.4)'deki Gauss yasasını her zaman sağlayan $\boldsymbol{A}$ vektör potansiyel ve Faraday yasasını sağlayan $\phi$ skaler potansiyel

$$
\boldsymbol{E}=-\boldsymbol{\nabla} \phi-\frac{\partial \boldsymbol{A}}{\partial t} \quad \boldsymbol{B}=\boldsymbol{\nabla} \times \boldsymbol{A}
$$

olacak şekilde tanımlanabilir (Jackson, 1999). Burada (1.8) ile verilen elektrik ve magnetik alanlar Maxwell denklemlerini değiştirmez yani invaryant bırakır. Doğal olarak bu potansiyellere matematiksel açıdan uygun fonksiyonlar gözüyle bakılabilir. Bununla birlikte potansiyellerin fiziksel anlamları oldukça önemlidir. Burada yine (1.8) ile verilen elektrik ve magnetik alanlar 


$$
\phi^{\prime}=\phi+\frac{\partial f(\boldsymbol{r}, t)}{\partial t} \quad \boldsymbol{A}^{\prime}=\boldsymbol{A}-\boldsymbol{\nabla} f(\boldsymbol{r}, t)
$$

skaler ve vektör potansiyel dönüşümleri altında invaryant kalır (Gasiorowicz, 1974). Elektrik ve magnetik alanı invaryant bırakan $(\phi, \boldsymbol{A}) \rightarrow\left(\phi^{\prime}, \boldsymbol{A}^{\prime}\right)$ dönüşüme ayar dönüşümü denir. Burada $f(\boldsymbol{r}, t)$ fonksiyonu dönüşüme aracı olan herhangi bir fonksiyondur ve dönüşüm fonksiyonu olarak bilinir. Ayar dönüşümleri altında EM alanlar değişmediği için klasik parçacığın hareketini anlatan (1.2) ile verilen denklemler invaryant kalır. Benzer şekilde kuantum mekaniğinde $P=|\psi|^{2}$ olasılığı ve gözlenebilir nicelikler de invaryant kalmalıdır (Peshkin ve Tonomura, 1989; Sakurai, 1994). Bu nedenle

$$
\psi^{\prime}=e^{i S / \hbar} \psi
$$

dönüşümü altında Schrödinger dalga denklemi de invaryant kalmalıdır (Sakurai, 1994). Burada $S(\boldsymbol{r}, t)$ yerel (local) fazdır. Bilindiği gibi serbest parçacık için Schrödinger dalga denklemi

$$
i \hbar \frac{\partial}{\partial t} \psi_{0}=\frac{(-i \hbar \nabla)^{2}}{2 m} \psi_{0}
$$

ile verilir ve

$$
i \hbar \frac{\partial}{\partial t} \rightarrow i \hbar \frac{\partial}{\partial t}-q \phi \quad-i \hbar \nabla \rightarrow-i \hbar \nabla-q \boldsymbol{A}
$$

operatör dönüşümlerinden $(\phi, \boldsymbol{A})$ potansiyeli ile etkileşen yüklü bir parçacığın

$$
\left(i \hbar \frac{\partial}{\partial t}-q \phi\right) \psi=\frac{(-i \hbar \nabla-q \boldsymbol{A})^{2}}{2 m} \psi
$$

Schrödinger dalga denklemi elde edilmektedir. Bu denklem $(\phi, \boldsymbol{A}, \psi) \rightarrow\left(\phi^{\prime}, \boldsymbol{A}^{\prime}, \psi^{\prime}\right)$ dönüşümleri altında ve $S(\boldsymbol{r}, t)=-q f(\boldsymbol{r}, t)$ koşulu sağlandığı sürece invaryant kalır. Ayrıca $\left(\phi^{\prime}, \boldsymbol{A}^{\prime}, \psi^{\prime}\right) \rightarrow\left(0,0, \psi_{0}\right)$ dönüşümü serbest parçacığın Schrödinger dalga denklemini vereceği için dalga fonksiyonu da $\psi^{\prime} \rightarrow \psi_{0}$ şeklinde dönüşeceğinden,

$$
\phi=-\frac{\partial f(\boldsymbol{r}, t)}{\partial t} \quad \text { ve } \quad \boldsymbol{A}=\boldsymbol{\nabla} f(\boldsymbol{r}, t)
$$

aynı zamanda sağlanmış olur. $f(\boldsymbol{r}, t)$ fonksiyonun

$$
d f(\boldsymbol{r}, t)=\frac{\partial f(\boldsymbol{r}, t)}{\partial t} d t+\nabla f(\boldsymbol{r}, t) \cdot d \boldsymbol{r}
$$

tam diferansiyelinde (1.14) ifadeleri (1.15)'da yerine yazıldıktan sonra her iki tarafin integrali alınarak $f(\boldsymbol{r}, t)$, dolayısıyla

$$
S(\boldsymbol{r}, t)=q \int \phi(\boldsymbol{r}, t) d t-q \int \boldsymbol{A}(\boldsymbol{r}, t) \cdot d \boldsymbol{r}
$$


elde edilir. Böylece, $S(\boldsymbol{r}, t)$ fonksiyonu $A^{\mu}=\left(\frac{\phi}{c}, \boldsymbol{A}\right)$ ve $x^{\mu}=(c t, \boldsymbol{r})$ dörtlü vektörleriyle

$$
S=q \int A^{\mu} d x_{\mu}
$$

şeklinde yazılabildiği için dalga fonksiyonu çözümleri

$$
\psi(x)=\psi_{0}(x) e^{-i q / \hbar \int A^{\mu} d x_{\mu}}
$$

potansiyeller cinsinden elde edilmiş olur (Gasiorowicz, 1974).

\section{Materyal ve Metot}

Başlangıçta düşünce (gedanken) deneyi olarak tasarlanan Aharonov-Bohm olayı ilk olarak 1961 yılında Robert G. Chambers, 1962 y1lında G. Möllenstedt ve W. Bayh, 1982 ve 1986 yılında A. Tonomura ve arkadaşları tarafından deneysel olarak doğrulanarak daha çekici hale gelmiştir (Chambers, 1960; Möllenstedt ve Bayh, 1962; Tonomura ve ark., 1982; Tonomura ve ark., 1986).

\subsection{Aharonov-Bohm Deneyi}

Kuantum parçacıklarıyla çift yarık deneyi yapılabilir ve kuantum mekaniksel olarak girişim olayı incelenebilir. Kuantum mekaniğinde dalga fonksiyonu konum, momentum ve enerji gibi dinamik değişkenlerin istatistiksel dağılımları hakkında bilgiler içeren soyut bir niceliktir. Potansiyel bölgesinde yayılan elektronlar $\psi_{1}=\psi_{01} e^{-i S_{1}}$ ve $\psi_{2}=\psi_{02} e^{-i S_{2}}$ dalga fonksiyonlarıyla temsil edildiğinde, $\psi=\psi_{1}+\psi_{2}$ olmak üzere, olasıllk

$$
P=|\psi|^{2}=\left|\psi_{01}\right|^{2}+\left|\psi_{02}\right|^{2}+2\left|\psi_{01}\right|\left|\psi_{02}\right| \cos [(p d \sin \theta-\Delta S) / \hbar]
$$

ile verilir (Sakurai, 1994). Yüklü parçacıkların EM potansiyellerle etkileşiminden kaynaklanan $\Delta S / \hbar$ faz farkı girişim desenini kaydırır. Faz farkının girişime etkisini görebilmek için iki ayrı deney önerilmektedir.

Birinci deneyde skaler potansiyel sadece zamanın bir fonksiyonu olarak değiștiğinde ve $\frac{\partial A}{\partial t}=0$ olduğunda, (1.8)'deki formüle göre elektrik alan sıfırdır. Bu nedenle skaler potansiyel içinde hareket eden elektronlar, elektrik alanla etkileşmez (Şekil 2.1). Şekil 2.1'de görülen deney düzeneğinde, faraday kafesi görevini yapan metal silindirlerden geçen elektronların faz fark1

$$
\Delta S=q \int_{0}^{t} \Delta \phi\left(t^{\prime}\right) d t^{\prime}=\int_{0}^{t} \Delta V\left(t^{\prime}\right) d t^{\prime}
$$

ile hesaplanabilir (Aharonov ve Bohm, 1959). Silindirlere farklı potansiyeller uygulandiğında faz farkı girişim desenini $\Delta S / \hbar$ kadar kaydırmış olur. Eşit potansiyeller uygulandı̆̆ında ise girişim yoktur. 


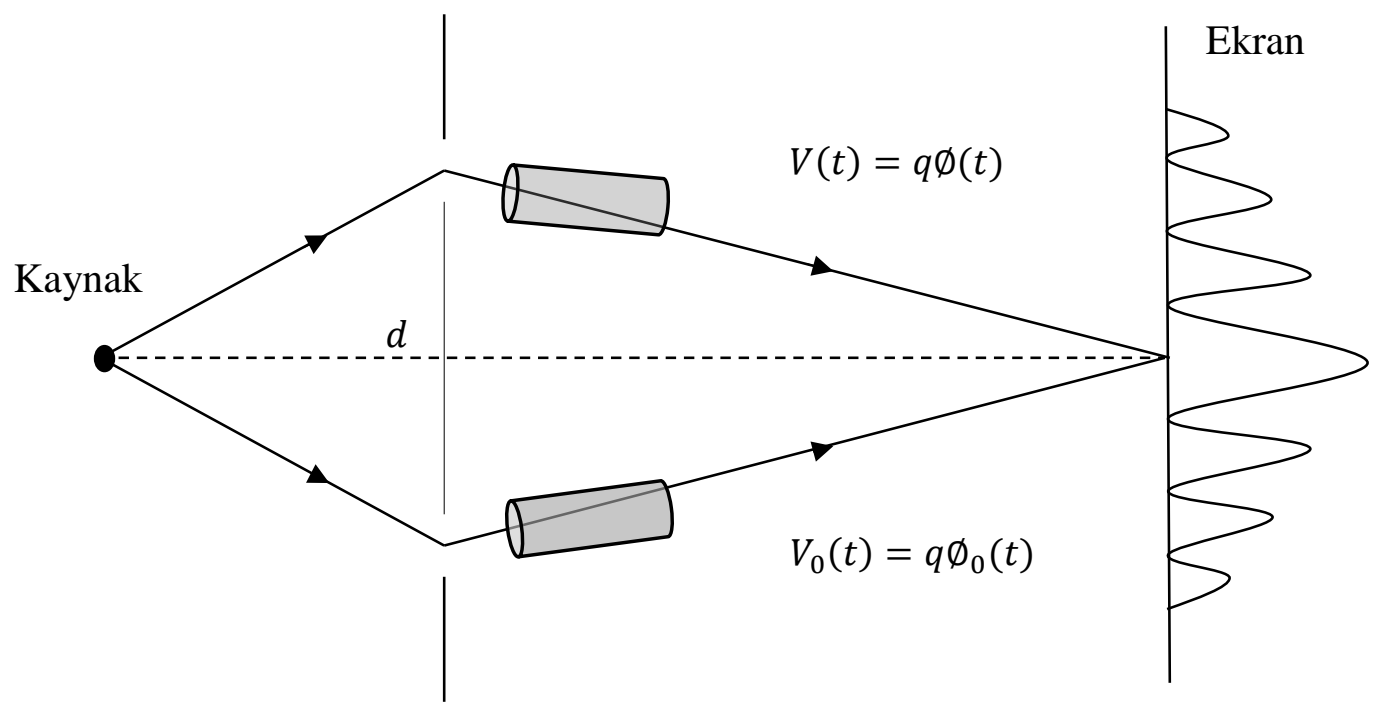

Şekil 2.1. Skaler potansiyel ile etkileşen elektronlar (Aharonov ve Bohm, 1959)

İkinci deneyde içinde düzgün magnetik alanın olduğu fakat dışında sıfır olduğu bir selenoid kullanılmaktadır (Şekil 2.2). Elektronlar, selenoidin dışında $\boldsymbol{B}=\boldsymbol{\nabla} \times \boldsymbol{A}=0$ şartını sağlayan vektör potansiyelle etkileşmektedir. Şekil 2.3'de selenoidin etrafından $C_{1}$ ve $C_{2}$ gibi farklı yolları takip eden elektronlar gösterilmektedir. Böylece, faz farkı

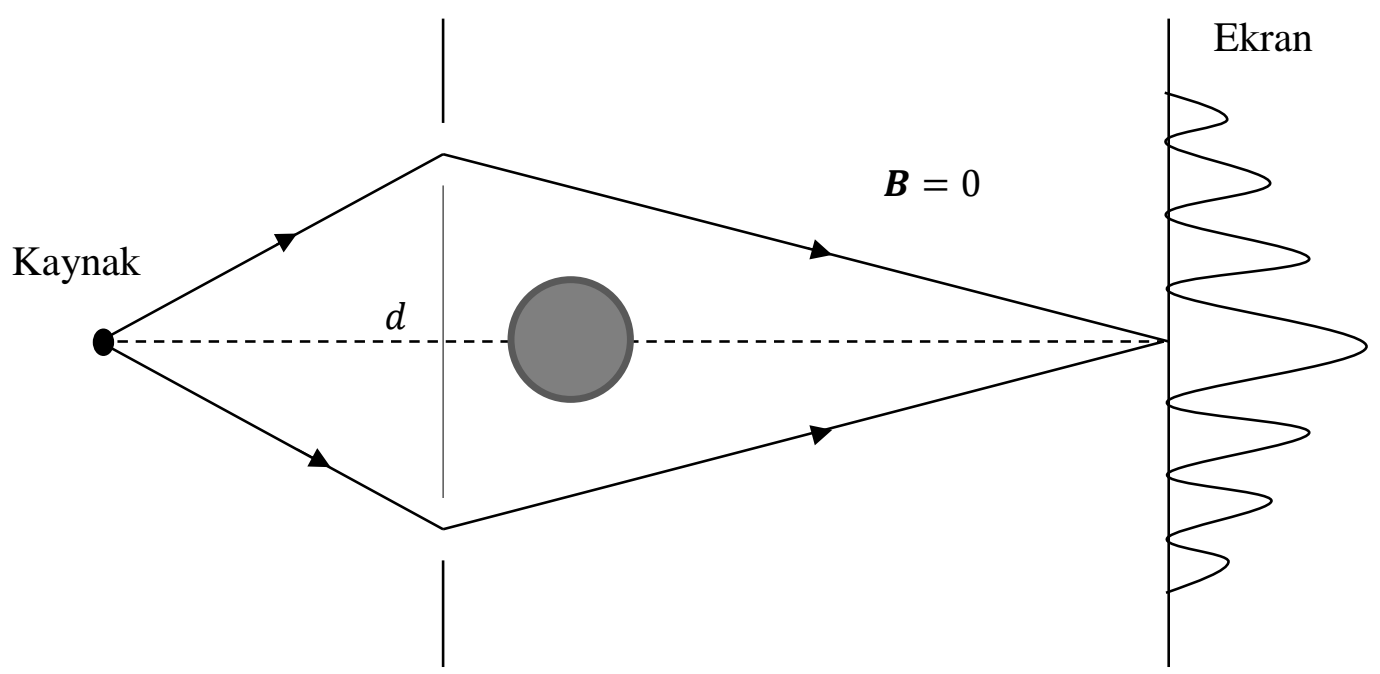

Şekil 2.2. Vektör potansiyel ile etkileşen elektronlar (Aharonov ve Bohm, 1959)

$$
\Delta S=-q \oint_{C} \boldsymbol{A}_{S} \cdot d \boldsymbol{r}=-q \oint_{S}\left(\boldsymbol{\nabla} \times \boldsymbol{A}_{S}\right) \cdot d \boldsymbol{a}=-q \int_{S} \boldsymbol{B}_{S} \cdot d \boldsymbol{a}=-q \phi_{S}
$$

gibi hesaplanır (Gasiorowicz, 1974). Şekil 2.3'de görüldüğü gibi $S$ yüzeyini $C$ eğrisi sınırlamaktadır. Burada $\Delta S$ fazı Stokes teoremine göre, kapalı bir eğri boyunca yol integralinin yüzey integraline çevrilmesiyle hesaplanır (Gasiorowicz, 1974). Burada faz farkının, selenoidin akısına bağlı olarak değiştiği ve girişim desenini $\Delta S / \hbar=-q \phi_{S} / \hbar$ kadar kaydırdığı görülmektedir. 


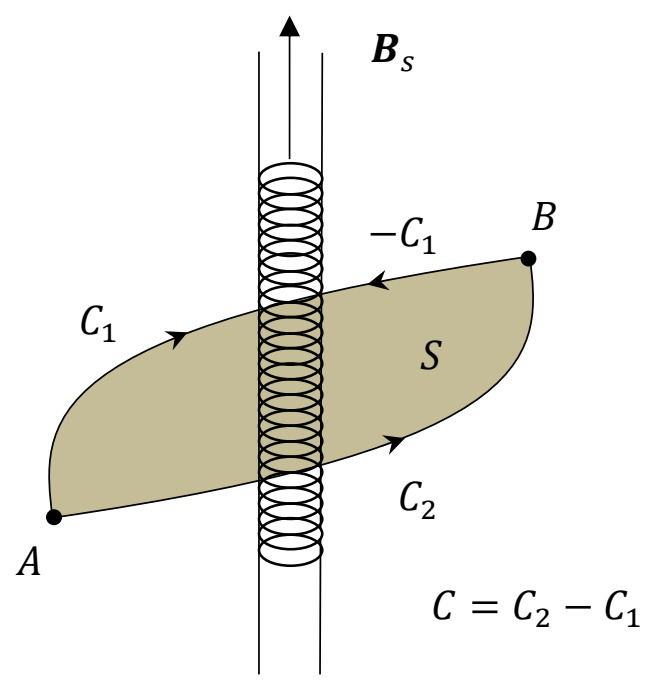

Şekil 2.3. $S$ yüzeyinden geçen selenoid ve bu yüzeyi sınırlayan $C$ eğrisi (Alvarez ve Hassan, 1997; Cheng and Li, 1984)

\section{Bulgular ve Tartışma}

Şimdiye kadar magnetik yüklerin veya tek-kutupların varlığı konusunda hiçbir deneysel kanıt bulunamamıştır. Tek-kutup fikri ilk defa 1931 yılında Paul Dirac tarafından ortaya atılmıştır (Dirac, 1931, 1948). Yüklerin kuantumlu oluşu fiziğin en derin gizlerinden biridir. Dirac'ın bu düşüncesi elektrik yüklerinin kuantumlu yapısına bir açıklama getirebilmektedir. Fizikçilerin ilgisini çeken Dirac'1n bu düşüncesi hala büyük bir çekiciliğe sahiptir.

\subsection{Simetrik Maxwell Denklemleri}

Boşlukta, genelleştirilmiş (simetrik) Maxwell denklemleri

$$
\begin{aligned}
& \boldsymbol{\nabla} \cdot \boldsymbol{E}=\frac{\rho_{e}}{\epsilon_{0}} \quad \boldsymbol{\nabla} \cdot \boldsymbol{B}=\mu_{0} \rho_{m} \quad \text { Gauss } \\
& \boldsymbol{\nabla} \times \boldsymbol{E}=-\frac{\partial \boldsymbol{B}}{\partial t}-\mu_{0} \boldsymbol{J}_{m} \quad \text { Faraday } \\
& \boldsymbol{\nabla} \times \boldsymbol{B}=\mu_{0} \boldsymbol{j}_{e}+\mu_{0} \epsilon_{0} \frac{\partial \boldsymbol{E}}{\partial t} \quad \text { Ampere-Maxwell }
\end{aligned}
$$

ve yüklü bir parçacık üzerine etkiyen Lorentz kuvveti

$$
\boldsymbol{F}=q_{e}(\boldsymbol{E}+\boldsymbol{v} \times \boldsymbol{B})+g\left[\boldsymbol{B}-(\boldsymbol{v} \times \boldsymbol{E}) / c^{2}\right]
$$

ile verilir (Jackson, 1999). Burada $\rho_{e}$ elektrik yük ve $\boldsymbol{j}_{\boldsymbol{e}}$ elektrik akım yoğunluğu olmak üzere, bunun gibi $\rho_{m}$ magnetik yük ve $\boldsymbol{j}_{\boldsymbol{m}}$ magnetik akım yoğunluğu tanımlandığında

$$
\frac{\partial \rho_{e}}{\partial t}+\boldsymbol{\nabla} \cdot \boldsymbol{j}_{\boldsymbol{e}}=0 \quad \frac{\partial \rho_{m}}{\partial t}+\boldsymbol{\nabla} \cdot \boldsymbol{j}_{\boldsymbol{m}}=0
$$

yük korunum yasaları da Maxwell denklemleri içinde kendiliğinden sağlanır. Buradaki (3.1)'den (3.5)'e kadar olan genelleştirilmiş Maxwell denklem takımı 


$$
\begin{array}{lll}
\boldsymbol{E} \rightarrow \boldsymbol{B} / \mu_{0} \epsilon_{0} & \boldsymbol{j}_{e} \rightarrow \boldsymbol{j}_{m} & \rho_{e} \rightarrow \rho_{m} \\
\boldsymbol{B} \rightarrow-\boldsymbol{E} & \boldsymbol{j}_{m} \rightarrow-\boldsymbol{j}_{e} / \mu_{0} \epsilon_{0} & \rho_{m} \rightarrow-\rho_{e} / \mu_{0} \epsilon_{0} \\
q_{e} \rightarrow g \mu_{0} \epsilon_{0} & \multicolumn{2}{c}{g \rightarrow-q_{e}}
\end{array}
$$

dönüşümleri altında simetriktir ve bu dönüşümlere duality dönüşümleri denir (Jackson, 1999). Bununla beraber, $\boldsymbol{E} \times \boldsymbol{B},(\boldsymbol{E} \cdot \boldsymbol{D}+\boldsymbol{B} \cdot \boldsymbol{H})$ ve $T_{\alpha \beta}$ EM gerilim tensörünün bileşenleri de invaryant kalır. Üslü kaynaklar $\left(\rho_{e}^{\prime}, \boldsymbol{j}_{e}^{\prime}, \rho_{m}^{\prime}, \boldsymbol{j}_{m}^{\prime}\right)$ ile yazılan ve üslü alan $\left(\boldsymbol{E}^{\prime}, \boldsymbol{B}^{\prime}, \boldsymbol{D}^{\prime}, \boldsymbol{H}^{\prime}\right)$ denklemleri simetrik Maxwell denklemleriyle aynıdır (Jackson, 1999).

\subsection{Dirac Monopolü}

Dirac monopolü ve kuantum koşulu Dirac sicim (string) kavramına dayanır. Şekil 3.1'deki gibi uç uca eklenmiş bir çift-kutuplar zincirinin, ya da sıkıca sarılmış selenoidin bir ucu olarak düşünülür ve diğer ucu sonsuza uzanır. Bu nedenle çift-kutuplar zincirine ya da selenoide sicim ad1 verilir. $\rho_{e}=q \delta(\boldsymbol{r})$ noktasal elektrik yükün $\boldsymbol{E}=K_{e} q \hat{\boldsymbol{r}} / r^{2}$ alanı gibi $\rho_{m}=g \delta(\boldsymbol{r})$ noktasal tek-kutup yükün $\boldsymbol{B}_{m}=K_{m} g \hat{\boldsymbol{r}} / r^{2}$ alanı tanımlanabilir. Burada, $K_{e}=\frac{1}{4 \pi \epsilon_{0}}=$ $910^{9} \mathrm{~N} \mathrm{~m}^{2} / \mathrm{C}^{2}$ ve $K_{m}=\frac{\mu_{0}}{4 \pi}=10^{-7} \mathrm{~T} \mathrm{~m} / \mathrm{A}$ ile tanımlanan sabitlerdir ve $K_{e} / K_{m}=1 / \epsilon_{0} \mu_{0}=$ $c^{2}$ olup burada $c$ ş̧ık hızıdır.

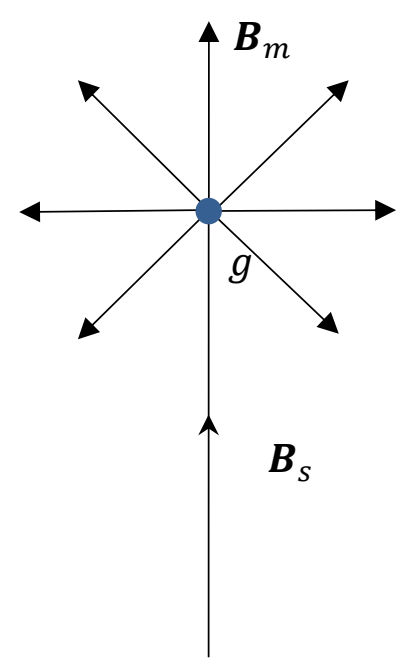

Şekil 3.1. Dirac sicim ve monopol sistemi (Alvarez ve Hassan, 1997; Cheng and Li, 1984)

Simetrik Maxwell denklem takımında (3.1) ile verilen $\boldsymbol{\nabla} \cdot \boldsymbol{B}_{m}=4 \pi K_{m} \rho_{m} \neq 0$ olduğu için $\boldsymbol{B}_{m}=\boldsymbol{\nabla} \times \boldsymbol{A}_{m}$ eşitliğini sağlayan herhangi bir $\boldsymbol{A}_{m}$ vektör potansiyeli bulunamaz. Dirac bu problemi ortadan kaldırabilmek için $(0,0,-\infty)$ ve $(0,0,0)$ aralığında yarı-sonsuz yapay bir selenoid önerdi (Dirac, 1931, 1948). Artık tek-kutup ve ona bağlı sicim az çok doğal olarak alışılmış EM etkileşmeler çerçevesi içinde işleme sokulabildiği için (1.8)'deki $\boldsymbol{B}=\boldsymbol{\nabla} \times \boldsymbol{A}$ ifadesi kullanılabilir. Doğada henüz magnetik tek-kutup olmadığına göre, (1.4)'deki Gauss yasası sağlanacak şekilde magnetik alanın tek-kutup ve yarı-sonsuz selenoid sisteminden oluştuğu düşüncesiyle ve $\boldsymbol{B}=\boldsymbol{B}_{m}+\boldsymbol{B}_{s}$ olmak üzere,

$$
\boldsymbol{\nabla} \cdot \boldsymbol{B}=\boldsymbol{\nabla} \cdot\left(\boldsymbol{B}_{m}+\boldsymbol{B}_{s}\right)=0
$$


eşitliği sağlanır (Şekil 3.1). Burada, $\boldsymbol{\nabla} \cdot\left(\frac{\hat{r}}{r^{2}}\right)=4 \pi \delta(\boldsymbol{r})=4 \pi \delta(x) \delta(y) \delta(z)$ olduğundan, monopol ve selenoid alanın uzaysal türevleri

$$
\boldsymbol{\nabla} \cdot \boldsymbol{B}_{m}=-\boldsymbol{\nabla} \cdot \boldsymbol{B}_{s}=4 \pi K_{m} g \delta(\boldsymbol{r})=4 \pi K_{m} g \delta(x) \delta(y) \delta(z)
$$

ile verilir. Yar1-sonsuz selenoidin magnetik alanı

$$
\boldsymbol{B}_{s}=4 \pi K_{m} g \delta(x) \delta(y) \mathrm{H}(-z) \hat{\mathbf{z}}
$$

ile verildiğinde, Heaviside basamak fonksiyonun

$$
\mathrm{H}(z)=\int_{-\infty}^{z} \delta(s) d s \quad \frac{d \mathrm{H}(-z)}{d z}=-\delta(z)
$$

tanımı kullanılarak (3.8) eşitliği sağlanır (Gradshteyn ve Ryzhik, 2000). Bu nedenle Şekil 3.1'deki tek-kutup ve sicim sisteminde

$$
\boldsymbol{B}=\frac{K_{m} g \hat{\boldsymbol{r}}}{r^{2}}+4 \pi K_{m} g \delta(x) \delta(y) \mathrm{H}(-z) \hat{\mathbf{z}}
$$

alanı (1.4)'deki Gauss yasasını sağlamaktadır (Alvarez ve Hassan, 1997; Cheng and Li, 1984).

Dirac'a göre: Elektronun uzun ince selenoid ile değil de, magnetik tek-kutupla olan etkileşmesinin betimlenebilmesi için elektronun tekil (singüler) $\boldsymbol{B}_{S}$ alanını hiçbir zaman "görmemesi" sağlanmalıdır. Dirac stringi yapay olduğu için gözlenemez ve fiziksel herhangi bir katkısı yoktur. Öyleyse elektronun dalga fonksiyonu sicim boyunca sıfır olmalıdır. Dirac daha sonra 1948 yılında bir makale yayınlayarak sicimlerin gözlenemezliğini ayrıntılı biçimde açıklamıştır (Dirac, 1931, 1948; Jackson, 1999).

$\boldsymbol{A}_{m}$ ve $\boldsymbol{A}_{s}$ potansiyelindeki tekillik birbirlerini yok edecek şekilde tek-kutup ve selenoid sistemi için singüler olmayan $\boldsymbol{A}=\boldsymbol{A}_{m}+\boldsymbol{A}_{s}$ potansiyeli tanımlanabilir (Alvarez ve Hassan, 1997; Cheng and Li, 1984).

Şekil 2.3 'deki $A$ noktasında dalga fonksiyonu $\psi_{0}$ verilmiş olsun. $A$ noktasından $C_{1}$ yolu üzerinden $B$ noktasına gidildiğinde faz ve dalga fonksiyonu

$$
S_{1}\left(c_{1} \text { yolu }\right)=-q \int_{A}^{B} \boldsymbol{A} \cdot \boldsymbol{d} \boldsymbol{r} \quad \text { ve } \quad \psi_{1}=\psi_{0} e^{-i S_{1}}
$$

ile ve $A$ noktasından $C_{2}$ yolu üzerinden $B$ noktasına gidildiğinde faz ve dalga fonksiyonu

$$
S_{2}\left(c_{2} \text { yolu }\right)=-q \int_{A}^{B} \boldsymbol{A} \cdot \boldsymbol{d} \boldsymbol{r} \quad \text { ve } \quad \psi_{2}=\psi_{0} e^{-i S_{2}}
$$

ile tanımlanmış olsun. $\mathrm{Bu}$ durumda fonksiyonların tek değerli olması veya girişim olayına herhangi bir katkıda bulunmaması için 


$$
e^{i \Delta S / \hbar}=1 \quad \text { ve } \quad \frac{\Delta S}{\hbar}=2 \pi N, \quad N \in \mathbb{Z}
$$

şartının sağlanması gerekir. (2.3) ifadesi (3.14)'de yazılarak

$$
\frac{q \phi_{s}}{\hbar}=2 \pi N, \quad N \in \mathbb{Z}
$$

koşulu yerine getirilir. Buradaki (3.15) koşuluna Dirac kuantum koşulu denir. Böylece (3.9)'daki alandan faydalanarak hesaplanan akının $\phi_{s}=\mu_{0} g$ değeri (3.15)'de yerine yazıldığında,

$$
2 K_{m} q g=N \hbar
$$

Dirac'ın yük kuantum koşulu elde edilmiş olur (Dirac, 1931, 1948). Gerçekten de gördüğümüz gibi, Schrödinger denkleminin ayar değişmezliği ve dalga fonksiyonun tekdeğerlilik gereksinimleri, Dirac'ın kuantum koşuluna yol açmaktadır (Jackson, 1999).

$N=1$ alındığgnda, Dirac'ın en temel magnetik yük birimi $g_{D}=\frac{\hbar}{2 K_{m} q}=\frac{q \cdot c}{2 \alpha}=3.310^{-9} \mathrm{~A} \cdot \mathrm{m}$ elde edilir. Böylece, $g=N g_{D}$ olmak üzere, magnetik yükler Dirac'in en temel magnetik yük biriminin tam katları olacak şekilde kuantumlanır. Benzer şekilde $N=1$ alındığında, Dirac'ın en temel elektrik yük birimi $q_{D}=\frac{\hbar}{2 K_{m} g_{D}}=1.610^{-19} \mathrm{~A} \cdot \mathrm{s}$ elde edilir. Buradan bildiğimiz standart elektrik yükleri $q=N q_{D}$ olmak üzere en temel elektrik yük biriminin tam katları şekilde kuantumlanmış olur. İnce-yapı sabiti $\alpha=\frac{e^{2}}{4 \pi \epsilon_{0} \hbar c} \sim \frac{1}{137}$ gibi bir magnetik ince-yapı sabiti $\alpha_{m}=\frac{g^{2}}{4 \pi \mu_{0} \hbar c}$ tanımlanırsa $g=\frac{N e c}{2 \alpha}$ magnetik yükü, $\alpha$ ince-yap1 sabiti cinsinden verilebilir. Böylece, $g^{2} \cong \frac{e^{2}}{4 \alpha^{2}}$ ifadesinden iki monopol arasındaki magnetik çiftlenimin $10^{4}$ kere elektriksel çiftleniminden daha büyük olduğu görülür.

Elektronun klasik yarıçapı $r_{e}=\frac{K_{e} e^{2}}{m_{e} c^{2}}$ 'nin kuantum yarıçapı $\lambda_{e}=\frac{h}{m_{e} c}$ 'ya oranı $\frac{r_{e}}{\lambda_{e}}=\frac{\alpha}{2 \pi} \sim \frac{1}{860}$ bulunur. Buradan, elektronun klasik yarıçapının, kuantum yarıçapından küçük $\left(r_{e}<\lambda_{e}\right)$ olduğu görülür. Benzer şekilde bir monopolün $r_{m}=\frac{K_{m} g^{2}}{m_{g} c^{2}}$ klasik yarıçapının, $\lambda_{m}=\frac{h}{m_{g} c}$ kuantum yarıçapına oranından $\frac{r_{m}}{\lambda_{m}}=\frac{N^{2}}{8 \pi \alpha} \sim 5.45 N^{2}$ bulunur. Buradan da monopolün klasik yarıçapının, kuantum yarıçapından büyük $\left(r_{m}>\lambda_{m}\right)$ olduğu görülmektedir.

\subsection{Yarı-Klasik Yöntem ile Kuantizasyon}

Orijinde durgun olan $g$ yüklü monopolün $\boldsymbol{B}=K_{m} g \boldsymbol{r} / r^{3}$ alanında, düşük hızlarda hareket eden $q$ yüklü parçacığa etkiyen kuvvet $\boldsymbol{F}_{B}=q \boldsymbol{v} \times \boldsymbol{B}$, orbital açısal momentum $\boldsymbol{L}=\boldsymbol{r} \times \boldsymbol{p}$ ve açısal momentumun zamanla değiş̧imi, $\frac{d \boldsymbol{L}}{d t}=\boldsymbol{\tau}_{\text {ext }}+\boldsymbol{\tau}_{B}$ ile tanımlanır. Burada, $\boldsymbol{\tau}_{\text {ext }}$ sisteme dışarıdan etkiyen ve diğer terim de magnetik alandan kaynaklanan $\boldsymbol{\tau}_{B}=\boldsymbol{r} \times \boldsymbol{F}_{\boldsymbol{B}}$ kuvvet momentidir. Bu tanımların ışığında açısal momentumun zamanla değiş̧imi 


$$
\frac{d \boldsymbol{L}}{d t}=\boldsymbol{\tau}_{\text {ext }}+K_{m} q g\left(\frac{\boldsymbol{v} r^{2}-(\boldsymbol{r} \cdot \boldsymbol{v}) \boldsymbol{r}}{r^{3}}\right)
$$

ile verilir (Alvarez ve Hassan, 1997). Monopolün kütlesinin $q$ yüklü parçacin kütlesine göre büyük olduğu varsayılmaktadır. Böylece, $q$ yükünün konumu $\boldsymbol{r}=x \widehat{\boldsymbol{x}}+y \widehat{\boldsymbol{y}}+z \hat{\boldsymbol{z}}$, hızı $\boldsymbol{v}=\dot{\boldsymbol{r}}=\dot{x} \hat{\boldsymbol{x}}+\dot{y} \hat{\boldsymbol{y}}+\dot{z} \hat{\boldsymbol{z}}$ ve $\boldsymbol{r} \cdot \boldsymbol{v}=x \dot{x}+y \dot{y}+z \dot{z}=r \dot{r}$ ifadeleri (3.17)'de yazilarak, $\frac{d J}{d t}=\boldsymbol{\tau}_{\text {ext }}$ denklemi elde edilir. Burada $\boldsymbol{J}=\boldsymbol{L}-K_{m} q g \hat{\boldsymbol{r}}$ toplam açısal momentum vektörüdür. $\boldsymbol{\tau}_{\text {ext }}=0$ olduğunda sistemin zamanla değişmeyen $\boldsymbol{J}$ toplam açısal momentum vektörü bulunur ve $\boldsymbol{J}_{e m}=-K_{m} q g \hat{\boldsymbol{r}}$ terimi alanlardan kaynaklanan EM açısal momentumdur (Alvarez ve Hassan, 1997).

Dirac'ın (3.16)'daki yük kuantum koşulu, yarı-klasik düşünce ile açıklanabilir. Bunun için

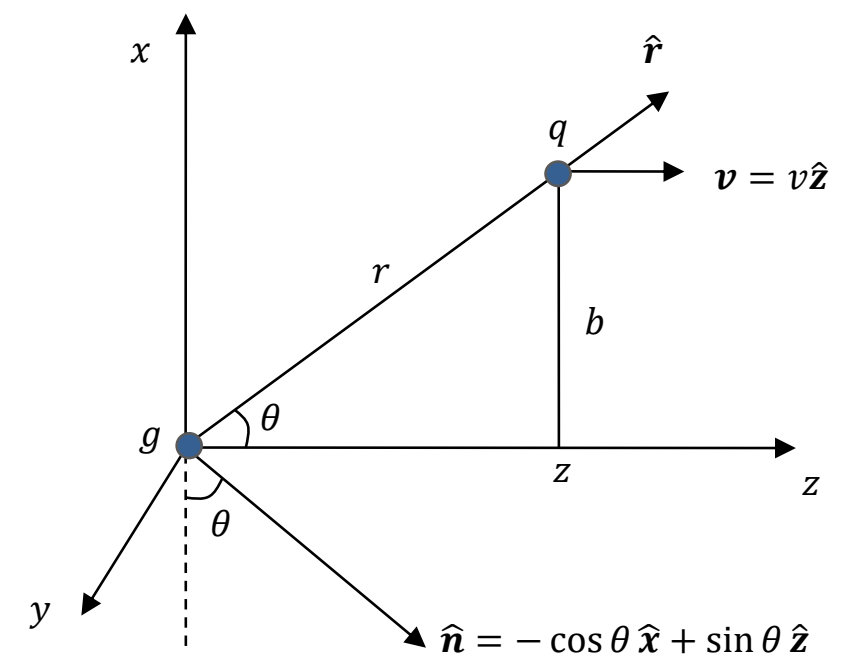

Şekil 3.2. Monopol alanından saçılan yüklü parçacık (Jackson, 1999)

Şekil 3.2'de görüldüğü gibi $q$ yüklü parçacık z-eksenine paralel olarak $b$ vurma parametresiyle ve $\boldsymbol{v}=\boldsymbol{v} \hat{\mathbf{z}}$ hızı ile gelmektedir. Bu parçacık üzerine monopolün $\boldsymbol{B}=$ $K_{m} g \boldsymbol{r} / r^{3}$ alanı 1şınsal olarak yönelmiştir ve Lorentz kuvvetince etkimektedir. Böylece çarpışma sürecinde ve sistemin yalıtılmış olduğu $\left(\boldsymbol{\tau}_{\text {ext }}=0\right)$ düşüncesiyle

$$
\frac{d \boldsymbol{L}}{d t}=\boldsymbol{\tau}_{B}=q v B b(-\cos \theta \hat{\boldsymbol{x}}+\sin \theta \hat{\mathbf{z}})
$$

ile verilir. Buradan

$$
\Delta \boldsymbol{L}=-\int_{-\infty}^{\infty} q v B b \cos \theta d t \widehat{\boldsymbol{x}}+\int_{-\infty}^{\infty} q v B b \sin \theta d t \hat{\boldsymbol{z}}
$$

integrali hesaplanarak bulunur. Yeterince büyük vurma parametrelerinde, yüklü parçacığın saptırılmadığını varsaydığımız yaklaşıklıkta $\frac{d x}{d t} \cong 0$ ve $\frac{d z}{d t} \cong v$ ifadeleri ve alanın $B=$ $K_{m} g /\left(z^{2}+b^{2}\right)$ değeri kullanılarak

$$
\Delta \boldsymbol{L}=\int_{-\infty}^{\infty}-\frac{q K_{m} g b z}{\left(z^{2}+b^{2}\right)^{3 / 2}} d z \hat{\boldsymbol{x}}+\int_{-\infty}^{\infty} \frac{q K_{m} g b^{2}}{\left(z^{2}+b^{2}\right)^{3 / 2}} d z \hat{\mathbf{z}}
$$


ifadesi elde edilir. İlk integral simetriden dolayı sıfirdır. Böylece, (3.20) integralinde $z=$ $b \cot \theta$ bağıntısı ile $\theta$ değişkenine geçilerek integral alındığında

$$
\Delta \boldsymbol{L}=2 K_{m} q g \hat{\mathbf{z}}
$$

elde edilir (Jackson, 1999).

Burada, açısal momentumun $z$-bileşenindeki değişim, yüklerin çarpımı ile orantılıdır ve yönü $g$ yükünden $q$ yüküne doğrudur. Yüklü parçacığın $v$ hızından ve $b$ vurma parametresinden bağımsızdır ve monopolü geçen parçacığın açısal momentumdaki değişim evrensel bir değerdir. Kuantum mekaniğine göre, açısal momentumun değişimi $\hbar$ planck sabitinin tam katları olmalıdır. Bu nedenle

$$
\Delta \boldsymbol{L}=2 K_{m} q g \hat{\mathbf{z}}=N \hbar \hat{\mathbf{z}} \quad N \in \mathbb{Z}
$$

gibi yazılabilir. Böylece, Dirac'in (3.16)'daki yük kuantum koşulu yarı-klasik yöntemle elde edilmiş olur (Jackson, 1999).

Ancak, burada açısal momentumun $z$-bileşeni korunmamaktadır. Halbuki sisteme dışarıdan herhangi bir tork etkimedikçe açısal momentum korunmalıdır. $\boldsymbol{\tau}_{\text {ext }}=0$ için $\boldsymbol{J}$ sabit bir vektördür ve $\boldsymbol{J}=\boldsymbol{L}+\boldsymbol{J}_{e m}$ gibi iki ayrı açısal momentumun toplamıdır. EM momentumun yoğunluğu $\boldsymbol{P}_{e m}=\epsilon_{0} \boldsymbol{E} \times \boldsymbol{B}$ ve EM açısal momentum vektörü

$$
\boldsymbol{J}_{\text {em }}=\int \boldsymbol{r} \times \boldsymbol{P}_{\text {em }} d \tau=\epsilon_{0} \int \boldsymbol{r} \times(\boldsymbol{E} \times \boldsymbol{B}) d \tau
$$

formülleri ilk defa J.J. Thomson tarafından önerilerek hesaplanmıştır (Jackson, 1999; Thomson, 1904a,b). (3.23) integralinin hesaplanabilmesi için, $g$ yüklü monopol orijinde ve $q$ yüklü parçacık monopolden $d$ kadar uzakta ve $z$ ekseninde olsun. Bu durumda, konum vektörleri arasındaki ilişki $\boldsymbol{r}^{\prime}=\boldsymbol{r}-\boldsymbol{d}$ ve $\boldsymbol{d}=d \hat{\mathbf{z}}$ olduğundan, alanlar

$$
\boldsymbol{E}=K_{e} q \boldsymbol{r}^{\prime} / r^{\prime 3} \quad \text { ve } \quad \boldsymbol{B}=K_{m} g \boldsymbol{r} / r^{3}
$$

ile verilir. Uzayda belirli bir noktanın tek-kutup yüküne uzaklığı $r=\sqrt{x^{2}+y^{2}+z^{2}}$ ve $q$ yüküne olan uzaklığı $r^{\prime}=r \sqrt{1+\frac{d^{2}}{r^{2}}-\frac{2 d \cos \theta}{r}}$ ile verilir. EM momentum yoğunluğunun, tüm uzay üzerinden integrali sıfırdır. Fakat toplam EM açısal momentumun değeri sıfır değildir. (3.24)'deki magnetik alanın değeri (3.23)'de yerine yazıldığında

$$
\boldsymbol{J}_{e m}=\epsilon_{0} K_{m} g \int\left(\frac{\boldsymbol{E}}{r}-\frac{\boldsymbol{r}(\boldsymbol{r} \cdot \boldsymbol{E})}{r^{3}}\right) d \tau
$$

elde edilir. Burada

$$
\boldsymbol{\nabla} \cdot\left(\frac{\boldsymbol{E} x_{i}}{r}\right)=\frac{x_{i}}{r}(\boldsymbol{\nabla} \cdot \boldsymbol{E})+\frac{E_{i}}{r}-\frac{x_{i} x_{j} E_{j}}{r^{3}}
$$

kullanılarak ve diverjans teoremi yardımıyla 


$$
J_{e m, i}=\epsilon_{0} K_{m} g \oint_{S} \frac{x_{i}}{r} \boldsymbol{E} \cdot d \boldsymbol{a}-\epsilon_{0} K_{m} g \int_{V} \frac{x_{i}}{r}(\boldsymbol{\nabla} \cdot \boldsymbol{E}) d \tau
$$

yüzey integraline çevrilir (Jackson, 1999). İlk terim, yükler arasındaki mesafenin, $S$ yüzey yarıçapıyla kıyaslandığında ihmal edilebilecek kadar küçük olduğu ve küresel simetriye sahip olduğu için sıfırdır. Böylece (3.27)'de $\boldsymbol{\nabla} \cdot \boldsymbol{E}=\rho_{e} / \epsilon_{0}$ ve $\rho_{e}=q \delta(\boldsymbol{r}-\boldsymbol{d})$ elektrik yük yoğunluğu yerine yazılarak, hacim üzerinden integral alındığında

$$
J_{\text {em }}=-K_{m} q g \boldsymbol{d} / d=-K_{m} q g \hat{\mathbf{z}}
$$

bulunur. (3.28)'deki EM açısal momentum vektörü yüklerin çarpımıyla orantılıdır ve yönü $q$ yükünden $g$ yüküne doğrudur. Eğer (3.28)'deki EM açısal momentum

$$
J_{e m}=-\frac{1}{2} N \hbar \hat{\mathbf{z}}, \quad N \in \mathbb{Z}
$$

şeklinde kesikli değerler alırsa, (3.28) ve (3.29) numaralı ifadelerin eşitliğinden, kuantum mekaniksel yol ile elde edilen $2 K_{m} q g=N \hbar$ Dirac kuantum koşulu elde edilir. Böylece, EM açısal momentum yarı-klasik bir düşünceyle kuantumlanmış olur.

Elektrik ve tek-kutup yükü taşıyan parçacığa dyon adı verilir ve $(q, g)$ ile gösterilir. $\left(q_{1}, g_{1}\right)$ ve $\left(q_{2}, g_{2}\right)$ gibi iki dyondan oluşan sistemin

$$
\boldsymbol{J}_{e m}=-K_{m}\left(q_{1} g_{2}-q_{2} g_{1}\right) \hat{\mathbf{z}}=\frac{N_{12}}{2} \hbar \hat{\mathbf{z}}
$$

açısal momentumu (3.29) kuantum koşuluna uyar ve (3.30)'daki ifade duality dönüşümleri altında invaryant kalır (Schwinger, 1966, 1968; Jackson, 1999).

\subsection{Ayar Dönüşümünün Anlamı}

Konumu $\boldsymbol{x}^{\prime}$ noktası boyunca yerleşmiş çift-kutuplar ya da sicimin uzayda belli bir $\boldsymbol{x}$ noktasında vektör potansiyele katkıları

$$
\boldsymbol{A}(\boldsymbol{x})=K_{m} g \int \boldsymbol{\nabla} \times\left(\frac{d \boldsymbol{x}^{\prime}}{\left|\boldsymbol{x}-\boldsymbol{x}^{\prime}\right|}\right)
$$

integrali ile verilir (Jackson, 1999). Bu integral hesab1 yapılırken geriye yalnızca sicimin yerleşimindeki keyfilik sorunu kalır. Açık olarak, fiziksel gözlenirler sicimin nerede bulunduğuna bağlı olmamalıdırlar. Değişik sicim konumlarının seçimi, vektör potansiyel için değişik ayar seçimlerine eşdeğerdir. Şekil 3.3'de görüldüğü gibi, $C_{1}$ ve $C_{2}$ gibi iki sicim düşünelim. $C_{1}$ eğrisi üzerinden alınan yol integrali ile elde edilen vektör potansiyel $\boldsymbol{A}_{+}$ve $C_{2}$ eğrisi üzerinden alınan yol integraliyle elde edilen vektör potansiyel de $\boldsymbol{A}_{-}$ile verilmiş olsun. Bunların arasındaki fark 


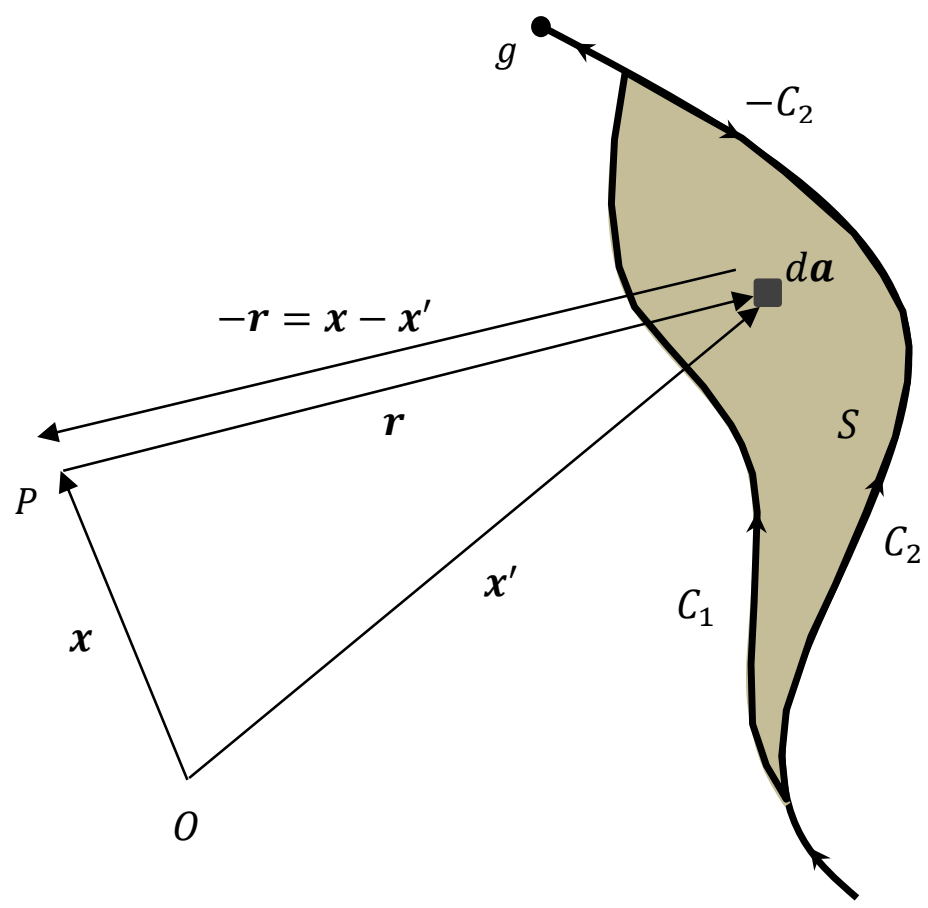

Şekil 3.3. $P$ gözlem noktasından $S$ yüzeyini gören katı açı $\Omega$ ve monopole uzanan sicimler (Jackson, 1999)

$$
\boldsymbol{A}_{+}-\boldsymbol{A}_{-}=K_{m} g \boldsymbol{\nabla} \times \oint_{\mathrm{C}} \psi d \boldsymbol{x}^{\prime}
$$

integraline eşittir. Burada hesaplarda kolaylık olması için $\psi=\frac{1}{\left|x-x^{\prime}\right|}$ skaler fonksiyonu tanımlanmıştır. Stokes teoreminin

$$
\oint_{C} \psi d \boldsymbol{x}=\oint_{S} d \boldsymbol{a} \times \nabla \psi
$$

tanımından (Jackson, 1999) faydalanarak $C$ kapalı yol integralinden $S$ yüzey integraline geçilebilir. Burada $\nabla^{\prime} \psi=-\nabla \psi, \nabla \times(\nabla \times \boldsymbol{A})=\boldsymbol{\nabla}(\boldsymbol{\nabla} \cdot \boldsymbol{A})-\boldsymbol{\nabla}^{2} \boldsymbol{A}$ operatör özdeşliklerinden ve $\nabla^{2}\left(\frac{1}{\left|x-x^{\prime}\right|}\right)=-4 \pi \delta\left(x-x^{\prime}\right)$ eşitliği kullanılarak,

$$
\boldsymbol{A}_{+}-\boldsymbol{A}_{-}=K_{m} g \nabla \Omega_{\mathrm{s}}+4 \pi K_{m} g \oint_{\mathrm{S}} \delta\left(\boldsymbol{x}-\boldsymbol{x}^{\prime}\right) d \boldsymbol{a}^{\prime}
$$

ifadesi bulunur. Burada

$$
\Omega_{\mathrm{S}}=\oint_{\mathrm{S}} \frac{-\left(\boldsymbol{x}-\boldsymbol{x}^{\prime}\right) \cdot d \boldsymbol{a}^{\prime}}{\left|\boldsymbol{x}-\boldsymbol{x}^{\prime}\right|^{3}}=\oint_{\mathrm{S}} \frac{\boldsymbol{r} \cdot d \boldsymbol{a}^{\prime}}{r^{3}}
$$

$P$ gözlem noktasından $S$ yüzeyini gören katı açıdır (Şekil 3.3). (3.34)'deki ikinci terimin katkısı, $\boldsymbol{x} \neq \boldsymbol{x}^{\prime}$ sıfır olduğundan nihayet potansiyellerin farkı,

$$
\boldsymbol{A}_{-}=\boldsymbol{A}_{+}-\boldsymbol{\nabla}\left(K_{m} g \Omega_{\mathrm{s}}\right)
$$


elde edilmiş olur. Böylece, (1.9) biçimindeki ayar dönüşümüyle karşılaştırıldığında sicimi $C_{1}$ 'den $C_{2}$ 'ye değiştirmenin $f=K_{m} g \Omega_{\mathrm{s}}$ fonksiyonlu bir ayar dönüşümüne eşdeğer olduğu görülür.

Kuantum mekaniğinde EM potansiyellerin ayarında yapılan bir değiştirmenin, dalga fonksiyonu

$$
\psi^{\prime}=e^{-i q f / \hbar} \psi=e^{-i K_{m} q g \Omega_{s} / \hbar} \psi
$$

uyarınca dönüştürmek koşuluyla, Schrödinger denkleminin biçimini değiş̧mez bıraktığı çok iyi bilinmektedir (Jackson, 1999). Buna göre, sicimin konumunda $C_{1}$ 'den $C_{2}$ 'ye olan bir değişmeye, elektronun dalga fonksiyonunun fazında bir düzeltme eşlik etmelidir, elektron $S$ yüzeyini geçerken $\Omega_{s}$ birdenbire $4 \pi$ kadar değiştiğinden, dalga fonksiyonun çok değerli olmaması için

$$
K_{m} q g \cdot 4 \pi / \hbar=2 \pi N \quad N \in \mathbb{Z}
$$

bağıntısının sağlanması gerekir (Jackson, 1999). Bunun sonucunda yine Dirac'ın kuantum koşulu elde edilir. Görüldüğü gibi, bu koşul, tek-kutup sicimin yerleşiminden bağımsız, ayar değişmezliği ve dalga fonksiyonunun tek değerli olması gibi gereksinimlerden çıkmaktadır (Sakurai, 1994; Wu T.T. ve Yang, 1975, 1976).

Magnetik tek-kutuplar için verilen bu tartışmalar, yalnızca en temel kavramları içermektedir. Kuantum koşullarının değiştirilmesi, magnetik tek-kutuplar ve elektrik yüklerini içeren kuramsal çalışmalar oldukça geniş bir konudur ve hala devam etmektedir (Alvarez ve Hassan, 1997; Schwinger, 1966, 1968; Preskill, 1984; Polchinski, 2004).

\subsection{Saçılma Olayı}

Burada, yüklü parçacıkların bir selenoid ile etkileşmesi sonucunda saçılma olayı incelenmektedir. Saçılma tesir kesitinin bulunabilmesi için gerekli olan dalga fonksiyonun çözümleri elde edilmektedir. Bu problemle ilgili deneysel düzenek Şekil 2.2'de verilmektedir. Kuantum mekaniğinden bildiğimiz gibi, potansiyel bölgesinde hareket eden yüklü bir parçacığın Schrödinger dalga denklemi (1.13) ile ve süreklilik denklemini sağlayan

$$
\boldsymbol{J}=\frac{\hbar}{2 m i}\left(\psi^{\star} \nabla \psi-\psi \nabla \psi^{\star}\right)-\frac{q}{m} \psi^{\star} \boldsymbol{A} \psi
$$

olasılık akım yoğunluğu ile verilir (Schiff, 1949). Bu problemi, vektör potansiyelin radyal bileşeni $A_{r}=0$ ve açısal bileşeni

$$
A_{\theta}(r)= \begin{cases}\frac{\Phi_{0} r}{2 \pi R^{2}}, & 0 \leq r \leq R \\ \frac{\Phi_{0}}{2 \pi r}, & R<r<\infty\end{cases}
$$

parçalı fonksiyonuyla verilen özel durum için çözelim. (1.13)'deki Schrödinger denklemi silindirik koordinatlarda 


$$
\left(\frac{\partial^{2}}{\partial r^{2}}+\frac{\partial}{r \partial r}+\frac{1}{r^{2}}\left(\frac{\partial}{\partial \theta}+i \alpha\right)^{2}+k^{2}\right) \psi(x)=0
$$

denklemine dönüşür (Aharonov ve Bohm, 1959). Burada, $k=\sqrt{2 m E / \hbar^{2}}$ serbest parçacı̆̆ın dalga vektörü ve

$$
\alpha=-q \Phi_{0} / 2 \pi \hbar
$$

akıyla orantılı olan boyutsuz bir niceliktir. Problem silindirik simetriye sahip olduğu için (4.3) denkleminin çözümü

$$
\psi(x)=\sum_{m=-\infty}^{\infty}\left[a_{m} J_{(m+\alpha)}(\rho)+b_{m} J_{-(m+\alpha)}(\rho)\right] e^{i m \theta}
$$

şeklinde $a_{m}$ ve $b_{m}$ gibi belli katsayılarla $J_{m+\alpha}(\rho)$ Bessel fonksiyonların çarpımından oluşan bir seri toplamla verilir. Bu çözümler $R$ yarıçaplı selenoidin dışında $(r>R)$ geçerlidir. Selenoidin içindeki $(r<R)$ çözümler de daha önceden bilinmektedir (Page, 1930). Her iki çözüm fonksiyonu ve türevleri $r=R$ sınırında sürekli olmalıdır (Yilmaz, 2014).

Alan bölgesinin dışında uzak bölgelerde (4.5) numaralı dalga fonksiyonun asimptotik davranışı

$$
\psi(x) \rightarrow e^{i k r \cos \theta} e^{-i \alpha \theta}+\frac{f(\theta) e^{i k r}}{\sqrt{2 \pi k r}} \sqrt{2 \pi k}
$$

gibi olacaktır (Schiff, 1949). Burada, ilk terimde Jacobi-Anger açılımı ve dalganın fazı

$$
e^{-i \alpha \theta}=\sum_{n=-\infty}^{\infty} \frac{\sin (n+\alpha) \pi}{(n+\alpha) \pi} e^{i n \theta} \quad-\pi<\theta<\pi
$$

seri açılımında Bessel fonksiyonların asimptotik davranışları kullanılarak

$$
\begin{aligned}
& \psi(x) \rightarrow \frac{e^{-i k r}}{\sqrt{2 \pi k r}} \sum_{n=-\infty}^{\infty} \sum_{m=-\infty}^{\infty} \frac{\sin (n+\alpha) \pi}{(n+\alpha) \pi} i^{m-n} e^{i m \theta} e^{i \varphi_{m-n}} \\
& +\frac{e^{i k r}}{\sqrt{2 \pi k r}}\left(\sum_{n=-\infty}^{\infty} \sum_{m=-\infty}^{\infty} \frac{\sin (n+\alpha) \pi}{(n+\alpha) \pi} i^{m-n} e^{i m \theta} e^{-i \varphi_{m-n}}+\sqrt{2 \pi k} f(\theta)\right)
\end{aligned}
$$

dalga fonksiyonu elde edilir (Gradshteyn ve Ryzhik, 2000). Bu fonksiyon içeri ve dişarı ışınsal yönde yayılan dalgaların toplamıdır.

Problemi biraz basitleştirmek için, selenoidin içindeki magnetik akının değerini sabit tutup, selenoid yarıçapının $R \rightarrow 0$ limit durumunu ele alalım. Selenoidin $R$ yarıçapı sıfıra gittiğinde, magnetik alan bölgesinde parçacığın bulunma olasılığı da sıfıra gideceği için $\psi(x)$ dalga fonksiyonu da sıfıra gider. $\mathrm{Bu}$ nedenle, (4.5) ile verilen genel çözümde, $R \rightarrow 0$ limit durumunda $b_{m}$ katsayıları sıfırdır. Böylece, bu yaklaşımda çözümler 


$$
\psi(x)=\sum_{m=-\infty}^{\infty} c_{m} J_{|m+\alpha|}(\rho) e^{i m \theta}
$$

ile verilir ve dalga fonksiyonuna pozitif mertebeli Bessel fonksiyonlar katkıda bulunur (Aharonov ve Bohm, 1959). Bessel fonksiyonların asimptotik davranışları, (4.9)'daki çözümde yerine yazılırsa

$$
\psi(x) \rightarrow \frac{e^{-i k r}}{\sqrt{2 \pi k r}} \sum_{m=-\infty}^{\infty} c_{m} e^{i m \theta} e^{i \varphi_{|m+\alpha|}}+\frac{e^{i k r}}{\sqrt{2 \pi k r}} \sum_{m=-\infty}^{\infty} c_{m} e^{i m \theta} e^{-i \varphi_{|m+\alpha|}}
$$

içeri ve dışarı ışınsal yönde yansıyan dalgaların toplamı olacaktır. (4.8)'deki dalga fonksiyonun yansıyan kısmı ile (4.10)'daki dalganın yansıyan kısımları birbirlerine eşitlenip diklik bağıntısı kullanılarak,

$$
c_{m}=e^{-i \varphi_{|m+\alpha|}} \sum_{n=-\infty}^{\infty} \frac{\sin (n+\alpha) \pi}{(n+\alpha) \pi} i^{m-n} e^{i \varphi_{m-n}}
$$

katsayıları bulunur. Yine (4.8)'deki dalga fonksiyonun 1şınsal doğrultuda ilerleyen kısmı ile (4.10)'daki dalganın ışınsal doğrultuda ilerleyen kısımları birbirlerine eşitlenip $c_{m}$ katsayıları yerine yazılarak

$$
f(\theta)=\frac{\sin (\pi \alpha)}{\sqrt{2 \pi i k}} \cdot \frac{e^{i \pi \alpha} e^{i([\alpha]+1 / 2) \theta}}{\sin (\theta / 2)}
$$

elde edilir. Burada $[\alpha]$, sayının tam kısmını ifade etmektedir. Böylece (4.12)'deki genlik (4.6)'da yerine yazıldığında

$$
\psi(x) \rightarrow e^{i k r \cos \theta} e^{-i \alpha \theta}+\frac{\sin (\pi \alpha)}{\sqrt{2 \pi i k r}} \cdot \frac{e^{i \pi \alpha} e^{i([\alpha]+1 / 2) \theta}}{\sin (\theta / 2)} \cdot e^{i k r}
$$

dalga fonksiyonu ve

$$
\frac{d \sigma}{d \theta}=|f(\theta)|^{2}=\frac{\sin ^{2}(\pi \alpha)}{2 \pi k} \frac{1}{\sin ^{2}(\theta / 2)}
$$

saçılmanın diferansiyel tesir kesiti hesaplanmış olur (Aharonov ve Bohm, 1959).

Burada eğer, $\alpha=N \in \mathbb{Z}$ ise $\sin (\pi \alpha)=0$ 'dır ve bu durumda saçılma tesir kesiti sıfırdır. $\psi(x) \rightarrow e^{i k r \cos \theta} e^{-i \alpha \theta}$ dalga fonksiyonun girişime etkisi yoktur ve dalga fonksiyonun tek değerli olması için

$$
\psi(\theta+2 \pi)=\psi(\theta)=e^{i 2 \pi \alpha}=1
$$


şartı sağlanır. Böylece, $\alpha=-\frac{q \Phi_{0}}{2 \pi \hbar}=N$ eşitliğinde akının $\Phi_{0}=4 \pi K_{m} g$ değeri yerine yazıldığında, (3.16)'daki Dirac'ın yük kuantum koşulu $2 K_{m} q g=N \hbar$ elde edilir (Dirac, 1931, 1948; Jackson, 1999).

Eğer $\alpha=N+\frac{1}{2}, N \in \mathbb{Z} \quad$ ise $\sin (\pi \alpha)=\cos (\pi N)=(-1)^{N} \quad$ ve $\quad$ (4.14)'den saçılmanın diferansiyel tesir kesiti

$$
\frac{d \sigma}{d \theta}=\frac{1}{2 \pi k} \cdot \frac{1}{\sin ^{2}(\theta / 2)}
$$

bulunur. $\mathrm{Bu}$ durumda girişimin maksimum olduğunu ve akının $\Phi_{0}=\frac{2 \pi \hbar}{q}\left(N+\frac{1}{2}\right)$ gibi kuantumlandığını görürüz.

\section{Sonuç}

Klasik mekaniğe göre yüklü parçacıklar alanlarla etkileşir. AB olayına göre alanlar olmasa bile parçacıklar potansiyellerle etkileşebilmektedir. Düşünce deneyi olarak ortaya çıkan AB olayı Tonomura ve arkadaşları tarafından deneysel olarak doğrulanmıştır. Bu olayın faz kayması, magnetik akı, magnetik tek-kutup kuantizasyonu gibi önemli sonuçları vardır.

Modern kuantum dünyasında $\mathrm{AB}$ olayı, Berry fazı, Dirac kuantizasyonu ve açısal momentum kuantizasyonu gibi konular, ayar dönüşümlerini kapsayacak şekilde birbirleriyle fiziksel açıdan sıkı bir iliş̧i içindelerdir (Sakurai, 1994).

Monopol Fikri ilk olarak 1931 yılında Paul Dirac tarafından ortaya atıldı. Şimdiye kadar magnetik yüklerin varlığı konusunda deneysel kanıt olmayışı, fizikçilerin monopole olan ilgisini azaltmaya yetmemiştir. Örneğin Georgi-Glashow, 't Hooft- Polyakov ve WeinbergSalam-Ward gibi birçok iddialı teorik model vardır.

Maxwell denklemlerinde elektrik yükü gibi, magnetik yükün olmaması ilginçtir. Ayar dönüşümleri sonucu invaryant kalan Maxwell denklemleri, Dirac'ın tek kutup düşüncesi ile de genelleştirilebilir. Eğer doğada magnetik tek kutup bulunuyorsa Dirac'ın yük kuantum koşuluna uymalıdır. Bu koşul bir elektronun elektrik yükü ile muhtemel bir monopolün yükünün çarpımının Planck sabitinin daima tam katı olması gerektiğini söyler. Böylece bu koşul doğadaki yüklerin, en temel yük biriminin niçin tam katları olması gerektiğine açıklama getirir.

Monopollerle ilgili ilk çalışmalardan biri olan Georgi-Glashow modeline göre monopolün kütlesi, protonun kütlesinin 8000 katıdır. Bunun gibi büyük birleşim teorilerinde, magnetik monopolün hesaplanan kütlesi yaklaşık olarak $10^{18} \mathrm{GeV} / \mathrm{c}^{2}$ mertebesindedir bu nedenle günümüz hızlandırıcılarında bu kütlenin gözlenmesi şimdilik mümkün değildir.

\section{Teşekkür}

UNSW Australia University, Faculty of Science, Theorical Physics Department araştırmacılarından Sayın Celal Harabati’ye, tartışma ve önerileriyle makalemize yaptı̆̆ değerli katkıları için sonsuz teşekkürlerimizi sunarız. 


\section{Kaynakça}

Aharonov Y., Bohm D., 1959. Significance of Elektromagnetic Potentials in the Quantum Theory. Physical Review 115: 485.

Alvarez-Gaume L., Hassan S.F., 1997. Introduction to S-Duality in N=2 Supersymmetric Gauge Theories. Fortsch. Phys. 45: 159-236.

Chambers R.G., 1960. Shift of an Electron Interference Pattern by Enclosed Magnetic Flux. Physical Review Letters 5: 3.

Dirac P.A.M., 1931. Quantised Singularities in the Electromagnetic Field. Proceedings of the Royal Society of London A 133: 821.

Dirac P.A.M., 1948. The Theory of Magnetic Poles. Physical Review 74: 817.

Gasiorowicz S., 1974. Quantum Physics. John Wiley and Sons, Singapore. 514 p.

Gradshteyn I. S., Ryzhik I.M., 2000. Table of Integrals, Series, and Products (6th ed.). Academic Press, United States of Amerika. 1163 p.

Goldstein H., Poole C., Safko J., 2002. Classical Mechanics (3rd ed.). Addision Wesley, United States of Amerika. 638 p.

Jackson J.D., 1999. Classical Electrodynamics (3rd ed.). John Wiley and Sons, Inc., New York. 808 p.

Möllenstedt G., Bayh W., 1962. Kontinuierliche Phasenschiebung von Elektronenwellen im kraftfeldfreien Raum durch das magnetische Vektorpotential eines Solenoids. Physikalische Blatter 18: 299-305.

Page L., 1930. Deflection of Electrons by a Magnetic Field on the Wawe Mechanics. Physical Review 36: 444.

Peshkin M., Tonomura A., 1989. The Aharonov - Bohm Effect (Lecture Notes in Physics). Springer - Verlag, Berlin Heidelberg. 153p.

Polchinski J., 2004. Monopoles, Duality, and String Theory. Int. J. Mod. Phys. A 19: 145156.

Preskill J., 1984. Magnetic Monopoles. Annual Review of Nuclear and Particle Science 34: 461-530.

Sakurai J.J., 1994. Modern Quantum Mechanics. Addison-Wesley Publishing Company, Inc., United States of America. 513 p.

Schiff L. I., 1949. Quantum Mechanics. McGraw-Hill Book Company, Inc., New York Toronto London. 417 p. 
Schwinger J., 1966. Magnetic Charge and Quantum Field Theory. Physical Review 144: 1087-1093.

Schwinger J., 1968. Sources and Magnetic Charge. Physical Review 173: 1536-1544.

Tai-Pei Cheng and Ling-Fong Li, 1984. Gauge Theory of Elemantary Particle Physics. Oxford University Press, New York. 536 p.

Thomson J.J., 1904. Elements of the Mathematical Theory of Electricity And Magnetism (3rd ed.). Cambridge University Press, England. 550 p.

Thomson J.J., 1904. On Momentum in the Electric Field. Philosophical Magazine 8: 331.

Tonomura A., Matsuda T., Suzuki R., Fukuhara A., Osakabe N., Umezaki H., Endo J., Shinagawa K., Sugita Y., Fujiwara H., 1982. Observation of Aharonov-Bohm Effect by Electron Holography. Physical Review Letters 48: 1443.

Tonomura A.,Osakabe N., Matsuda T., Kawasaki T., Endo J., Yano S., Yamada H., 1986. Evidence for Aharonov-Bohm Effect with Magnetic Field Completely Shielded from Electron Wawe. Physical Review Letters 56: 792.

Wu T. T., Yang C. N., 1975. Concept of nonintegrable phase factors and global formulation of gauge fields. Physical Review D 12: 3845.

Wu T. T., Yang C. N., 1976. Dirac monopole without strings: Monopole harmonics. Nuclear Physics B 107: 365-380.

Yilmaz O., 2014. Scattering of a Changed Particle from a Hard Cylindrical Selenoid: Aharonov-Bohm Effect. Chinese Journal of Physics 52: 1184-1198. 